\title{
A discrete particle swarm optimization algorithm with local search for a production-based two-echelon single-vendor multiple-buyer supply chain
}

\author{
Mehdi Seifbarghy $^{1} \cdot$ Masoud Mirzaei Kalani $^{2} \cdot$ Mojtaba Hemmati $^{2}$
}

Received: 3 August 2014/Accepted: 3 October 2015/Published online: 4 November 2015

(C) The Author(s) 2015. This article is published with open access at Springerlink.com

\begin{abstract}
This paper formulates a two-echelon singleproducer multi-buyer supply chain model, while a single product is produced and transported to the buyers by the producer. The producer and the buyers apply vendormanaged inventory mode of operation. It is assumed that the producer applies economic production quantity policy, which implies a constant production rate at the producer. The operational parameters of each buyer are sales quantity, sales price and production rate. Channel profit of the supply chain and contract price between the producer and each buyer is determined based on the values of the operational parameters. Since the model belongs to nonlinear integer programs, we use a discrete particle swarm optimization algorithm (DPSO) to solve the addressed problem; however, the performance of the DPSO is compared utilizing two well-known heuristics, namely genetic algorithm and simulated annealing. A number of examples are provided to verify the model and assess the performance of the proposed heuristics. Experimental results indicate that DPSO outperforms the rival heuristics, with respect to some comparison metrics.
\end{abstract}

Mehdi Seifbarghy

seifbar@yahoo.com; m.seifbarghy@alzahra.ac.ir

Masoud Mirzaei Kalani

masood.mirzaie@gmail.com

Mojtaba Hemmati

mojtaba_h86@yahoo.com

1 Department of Industrial Engineering, Alzahra University, Tehran, Iran

2 Faculty of Industrial and Mechanical Engineering, Qazvin Branch, Islamic Azad University, Qazvin, Iran
Keywords Vendor-managed inventory $\cdot$ Economic production quantity $\cdot$ Supply chain - Particle swarm optimization

\section{Introduction}

A supply chain consists of a number of organizations with materials, information and cash flows among them. Considering the first and last organizations as supplier and customer, respectively, the chain's objective is satisfying customer requirements with optimal operational cost. Vendor-managed inventory (VMI) as a modern IT-based partnership technique has been of great attention in recent years. In a VMI partnership, the supplier, usually the manufacturer but sometimes a reseller or distributor, makes the main inventory replenishment decisions for the consuming organization in such a way that the vendor monitors the buyer's inventory levels (physically or via electronic messaging) and makes periodic replenishment decisions.

This paper is an extension to Nachiappan and Jawahar (2007) in which a two-echelon single-producer multi-buyer supply chain (TSPMBSC) model while the vendor applies economic production quantity (EPQ) instead of economic order quantity (EOQ) is formulated. The producer and buyers apply VMI mode of operation. The production rate of the producer is assumed to be restricted. As an EOQ inventory control system, the production is done during a specific part of the replenishment cycle time. The buyers are assumed to employ the well-known EOQ inventory control system. The operational parameters are sales quantity, sales price and the production rate for each buyer which should be determined at the producer's location. Channel profit of the supply chain and contract price 
between the producer and buyers is determined based on the optimal values of the addressed operational parameters. A mathematical programming model is developed to find out the optimal values of the operational parameters. The model has a nonlinear objective function involving several integer variables and three different sets of linear constraints; it belongs to nonlinear integer programs (NIP). Considering Govindan (2013) which researches on VMI are classified into three categories of modeling, simulation, and case studies, we can conclude that this paper falls into the modeling category.

Costa and Oliveira (2001) addressed that the evolutionary strategies such as genetic algorithm (GA) and simulated annealing algorithm (SA) are emerging as the best algorithms for solving NIP problems. GA and SA could be useful for this NIP problem to provide near to optimal solutions.

The revenue sharing and the partnership among members of the supply chain are the major issues for the success of a supply chain. The net revenue is addressed as channel profit ' $P c$ ' which depends on sales quantity. Sales quantity is influenced by sales price (Waller et al. 2001). However, the formulations would show that it depends on both sales quantity and production rate while the vendor is also the producer. The relationship between sales quantity and sales price could be assumed to behave linearly (Lau and Lau 2003). It is generally believed that the pricing acceptable (fair) to the partners involved is an important factor to make constant relations in VMI, and that it requires acceptable revenue sharing that would satisfy both the vendor and the buyer (Grieger 2003). This reveals that the revenue sharing between the vendor and the buyer plays a vital role in determining the contract price.

The organization of the rest of the paper is as follows: Sect. 2 is on the literature review, problem description and modeling is given in Sect. 3. Section 4 gives the proposed heuristics to solve the problem. In Sect. 5, a number of numerical examples of different sizes are presented and solved to measure the accuracy of the proposed heuristics. Section 6 gives the research conclusions and ideas for further research.

\section{Literature review}

The literature on two-echelon supply chains is rich enough. Bhattacharjee and Ramesh (2000) developed two efficient heuristics to derive the optimal price and ordering policies to maximize the net profit of the retailer for a multi-period inventory and pricing model. Lu (1995) pointed out that future researches should consider the buyer's point of view and there should be a minimal acceptable profit level to both the vendor and buyers; this made a suitable base for the well-known concept which is revenue sharing. Maloni and Benton (1997) stated that the major focus of revenue sharing is to share the revenues/profits generated based on the assignments and responsibilities to avoid the conflict between supply chain partners. Yao and Chiou (2004) considered the single-vendor and multi-buyer model proposed by $\mathrm{Lu}$ (1995) and identified that the vendor's optimal annual cost function was a piecewise convex curve with respect to the vendors' production setup interval; they suggested that a search algorithm can be developed to obtain an optimal solution for a sub-problem. They also proposed a search algorithm and demonstrated that their algorithm reached a better result than Lu's search procedure.

Nachiappan and Jawahar (2007) formulated an integrated inventory model of a two-echelon single-vendor multiple buyers (TSVMBSC) under the VMI mode of operation to maximize the channel profit and to share the profit among the members involved assuming that both vendors and buyers follow EOQ conditions. The given model in this paper is an extension to the model given by Nachiappan and Jawahar (2007) assuming that the entrance rate of products to the vendor's location is bounded (i.e., EPQ conditions); in the new formulation, the optimal production rate for each buyer in the vendor's (i.e., producer's) location is determined as well as the optimal sales quantities and sales prices.

Zhang et al. (2007) presented an integrated VMI model for a single vendor and multiple buyers, where the vendor purchases and processes raw materials and then delivers finished items to the buyers. A joint relevant cost model is developed with constant production and demand rates under the assumption that buyers' ordering cycles may be different and that each buyer can replenish more than once in one production cycle. The main point of this research is that demand rate at all buyers is constant while it is determined as a function of the sales price in the current research. Yao et al. (2007) developed an analytical model that explores how important supply chain parameters affect the cost savings to be realized from collaborative initiatives as VMI. Van der Vlist et al. (2007) argue on the conclusions drawn from Yao et al. (2007). They express that the model ignores the costs of shipments from the supplier to the buyer and plans the incoming and outgoing flows at the supplier in a manner that overstates the inventory needed.

Toptal and Çetinkaya (2008) aimed to develop analytical and numerical results representing the system-wide cost improvement rates which are due to coordination. Revisiting a few basic researches, they consider generalized replenishment costs under centralized decision making. This research analyzes (1) how the counterpart centralized and decentralized solutions differ from each other, (2) under what circumstances their implications are similar, 
and (3) the effect of generalized replenishment costs of the system-wide cost improvement rates which are subject to coordination. Wang (2009) studied a decentralized supply chain consisting of a single manufacturer and a single distributor for a short lifecycle product with random yield and uncertain demand as in the semiconductor industry. Two scenarios for handling the business are considered. One scenario is the traditional supply chain arrangement, where the distributor is fully responsible for the inventory decision, whereas the manufacturer is fully responsible for the production decision. The other scenario is the VMI arrangement, where the manufacturer is fully responsible for the entire production and inventory decisions in the supply chain. The optimal production and inventory decisions under both scenarios are compared.

$\mathrm{Yu}$ et al. (2009a) discussed how the manufacturer (vendor) can take advantage of the information received from retailers for increasing his own profit using a Stackelberg game in a VMI system. The manufacturer produces a finished product and supplies it at the same wholesale price to multiple retailers. The retailers sell the product in independent markets at retail prices. Solution procedures are developed to find the Stackelberg game equilibrium which each enterprise is not interested in deviating from Yu et al. (2009b) investigated how a manufacturer and its retailers cooperate each other to find their individual optimal net profits considering product marketing (advertising and pricing) and inventory policies in an information-asymmetric VMI supply chain. The manufacturer produces and gives a single product at the same wholesale price to multiple retailers who sell the product in their independent markets at retail prices. The manufacturer determines its wholesale price, advertising investment, replenishment cycles for the raw materials and finished product, and backorder quantity to maximize the profit. Retailers in turn consider the replenishment policies and the manufacturer's promotion policies and determine the optimal retail prices and advertisement investments to maximize their profits.

Zavanella and Zanoni (2009) investigated the way how a particular VMI policy, known as Consignment Stock (CS), may represent a successful strategy for both the buyer and the supplier. The most radical application of CS may lead to the suppression of the vendor inventory, as this actor uses the buyer's store to stock its finished products. As a counterpart, the vendor will guarantee that the quantity stored in the buyer's store will be kept between a maximum and a minimum level, also supporting the additional costs eventually induced by stock-out conditions. The buyer will pick up from its store the quantity of material needed to meet its production plans and the material itself will be paid to the buyer according to the agreement signed. Wong et al. (2009) studied on how a sales rebate contract helped to achieve supply chain coordination. For this purpose, a model in the context of a two-echelon supply chain with a single supplier serving multiple retailers in VMI partnership is proposed. VMI facilitates the application of the sales rebate contract since information sharing in VMI partnership lets the supplier to obtain actual sales data in a timely manner and determine the rebate for retailers. The proposed model indicates that the supplier gains more profit with competing retailers than without as competition among the retailers lowers the prices and correspondingly increases demand. Bichescu and Fry (2009) analyzed decentralized supply chains, which followed continuous review $(Q, R)$ inventory policies considering VMI agreements where the supplier chooses the order quantity $Q$, and the retailer chooses the reorder point $R$. The effect of divisions of channel power on supply chain and individual agent performance is investigated by examining different game theoretic models. The results showed that VMI can result in considerable supply chain savings rather than traditional relationships; furthermore, the greatest system benefits from VMI arise in asymmetric channel power relationships.

Almehdawe and Mantin (2010) consider a supply chain consisting of a single capacitated manufacturer and multiple retailers. A Stackelberg game VMI framework under two scenarios is utilized. Initially, the traditional approach wherein the manufacturer is the leader is considered; in the second, one of the retailers acts as the dominant player of the supply chain. Darwish and Odah (2010) developed a model for a supply chain with a single vendor and multiple retailers under the VMI mode of operation. The developed model can easily describe supply chains with capacity constraints by selecting high penalty cost. Theorems are given to tackle the complexity of the model. Furthermore, an efficient algorithm is devised to find the global optimal solution. Wang et al. (2010) investigate a recent paper by Yao et al. (2007) and a critique by Van der Vlist et al. (2007). Both researches presented interesting arguments to show their valuable findings. However, their finding on the buyer's order sizes seems to conflict with each other. Revisiting both papers, they come to the conclusion that both papers are valid within the scopes and assumptions of their own studies. Guan and Zhao (2010) considered a single-vendor and a single-buyer supply chain and study contracts for a VMI program. They design a revenue sharing contract for vendor with ownership scenario, and a franchising contract for retailer with ownership scenario. Based on continuous review $(R, Q)$ policy, without consideration of order policy and related costs at the vendor site, it is indicated that one contract can perform satisfactorily while the other one is a perfect contract. Considering order policy and related costs at the vendor site, it is indicated that one contract can perform satisfactorily while 
the performance of the other one depends on the system parameters. Bookbinder et al. (2010) consider a vendor, which manufactures a single product sold to a retailer. Three scenarios are studied: independent decision making in which there is no agreement between the parties; VMI, whereby the vendor initiates orders on behalf of the retailer; and central decision making in which both vendor and retailer are controlled by the same corporate entity. Optimal solutions are obtained analytically for the retailer's order quantity, the vendor's production quantity, the parties' individual and total costs in the three scenarios. Those situations in which VMI is beneficial are recognized. Razmi et al. (2010) considered a buyer-supplier supply chain and compared the performance of the traditional and VMI system using the total inventory cost of the supply chain as the performance measure. The concept of extent point is introduced in which the difference between the total cost of both traditional and VMI systems is minimal. It is applied to investigate how increasing or reducing the key parameters changes the total cost of the two systems with respect to each other. Goh and Ponnambalam (2010) proposed a mathematical model to determine the optimal sales quantity, optima sales price, optimal channel profit and contract price between the vendor and buyer in TSVMBSC under the VMI mode of operation. All the parameters depend on the understanding of the revenue sharing between the vendor and buyers. A particle swarm optimization (PSO) was proposed to solve the problem. The solutions obtained from PSO were compared with the previous results reported in the literature. Pasandideh et al. (2010) developed a model for a two-level supply chain consisting of a single supplier and a single retailer studying the inventory management practices before and after implementation of VMI. This research explores the effect of important supply chain parameters on the cost savings realized from collaborative initiatives. The results indicate that the VMI implementation of EOQ model when unsatisfied demand is backlogged sometimes has the ability to reduce total costs of supply chains.

Pasandideh et al. (2011) developed an EOQ model for a two-level supply chain consisting of one supplier and one retailer in which unsatisfied demands are backordered, the supplier's storage is constrained and there is an upper bound on the number of orders. They assume that the supplier utilizes the retailer's information in decision making on the replenishments and supplies orders to the retailer according to $(R, Q)$ policy. A GA is proposed to find the order quantities and the maximum backorder levels, so that the total inventory cost of the supply chain is minimized. Shao et al. (2011) studied inventory and pricing policies in a non-cooperative supply chain with one supplier and several retailers under an information-asymmetric VMI environment. The supplier produces a product at the wholesale price and gives to the retailers. The retailers distribute the product in markets at retail selling prices. The demand rate for each independent market is a non-decreasing concave function of the marketing expenditures of both local retailers and the manufacturer, but a non-increasing and convex function of the retail selling prices. Wholesale price, marketing expenditure for supplier and retailers, replenishment cycles for the product and backorder quantity are determined in such a way as to maximize the total profit. Sana et al. (2011) present an integrated production-inventory model that is presented for supplier, manufacturer and retailer supply chain, considering perfect and imperfect quality items. This model considers the impact of business strategies such as the optimal order size of raw materials, production rate and unit production cost, and idle times in different sectors on the collaborating marketing system. An analytical method is employed to optimize the production rate and raw material order size for maximum expected average profit. An example is illustrated to study the behavior and application of the model. Pal et al. (2012a, b) present a production inventory model for various types of items where multiple suppliers, a manufacturer and the multiple non-competing retailers are the members of the supply chain. And each supplier supplies only one type of raw material to the manufacturer. The manufacturer produces a finished item by the combination of a certain percentage of the various types of raw materials. The manufacturer produces also multi-items and delivers them according to the demand of the different retailers. Finally, an integrated profit of the supply chain is optimized by optimal ordering lot sizes of the raw materials. A numerical example is provided to justify the proposed model.

Pal et al. (2012a, b) develop a multi-echelon supply chain model for multiple markets with different selling seasons. Here, two suppliers are involved to supply the raw materials to the manufacturer where the main supplier may face supply disruption after a random time and the secondary supplier is perfectly reliable but more expensive than the main supplier. In their article, the manufacturer produces a random proportion of defective items which are reworked after regular production and are sold in a lot to another market just after completion of rework. The retailer sells the finished products in different markets according to seasons. Finally, an integrated expected cost per unit product of the chain is minimized analytically by considering the lot-size ordered as a decision variable. An appropriate numerical example is also provided to justify the proposed model. Goh et al. (2012) solved TSVMBSC model proposed by Nachiappan and Jawahar (2007) utilizing PSO and a hybrid of GA and artificial immune system (GA-AIS). These two algorithms are evaluated for their solution quality in the addressed research. Cárdenas-Barrón et al. (2012) 
presented an alternative heuristic algorithm to solve the vendor management inventory system with multi-product and multi-constraint based on an EOQ model with authorized stock out. Stock-out cost is considered linear and fixed. Since the problem is a nonlinear integer programming, a heuristic algorithm is proposed to solve the problem.

Sadeghi et al. (2013) studied a multi-vendor multi-retailer single-warehouse supply chain under the VMI mode of operation with constrained space and annual number of orders for the warehouse. The objective was to find the order quantities along with the number of shipments received by retailers and vendors in such a way as to minimize the total inventory cost. Nia et al. (2013) developed a multi-product EOQ model under a VMI policy in a single-vendor single-buyer supply chain. Unsatisfied demands are backordered. A few constraints such as storage capacity, number of deliveries and order quantity are considered in the given model. Demand, available storage and total order quantity are considered as fuzzy numbers. An ant colony optimization algorithm along with GA is utilized to find a near-optimum solution. AriaNezhad et al. (2013) attempts to develop the retailer's inventory model with the effect of order cancellations during the advance sales period. The retailer announces a price discount program during advance sales period to promote his sales and also offers trade credit financing during the sales periods. The retailer availing trade credit period from his supplier offers a permissible delay period to his customers. The customer who gets an item is allowed to pay on or before the permissible delay period which is accounted from the buying time rather than from the start period of inventory sales. This accounts for significant changes in the calculations of interest payable and interest earned by the retailer. The retailer's total cost is minimized so as to find out the optimal replenishment cycle time and price discount policies through a solution procedure. The results derived in mathematical theorems are implemented in numerical examples, and sensitivity analyses on several inventory parameters are obtained.

Diabat (2014) considered a two-echelon single-vendor multi-buyer supply chain network operated under VMI policy and found the optimal sales. Hybrid genetic/simulated annealing algorithm is developed to deal with the problem. Rad et al. (2014) considered a two-echelon supply chain consisting of a single vendor and two buyers. The vendor gives a single product to both buyers at a finite production rate. A mathematical model for the integrated VMI policy is developed. Furthermore, solution algorithms are proposed to determine the optimal lot size and total inventory cost of the supply chain. The effect of key parameters such as buyer's demand and vendor's holding cost on lot size variation is also studied. Results show that greater reduction in the total cost of the supply chain can be obtained using VMI. Verma et al. (2014) proposed an alternative replenishment scheme allowing for different replenishment cycles for each retailer in the single-vendor multi-retailer supply chain under VMI partnership. Taleizadeh and Noori-daryan (2014) considered a decentralized three-layer supply chain including a supplier, a producer and arbitrary number of retailers. Retailers order from the producer who is replenished by the supplier. Demand is assumed to be price sensitive. The paper optimizes the total cost of the supply chain network integrating decisionmaking policy using Stackelberg-Nash equilibrium. The decision variables of the model are the supplier's price, the producer's price and the number of shipments received by the supplier and the producer. Pasandideh et al. (2014a) studied single-vendor single-buyer supply chain system under VMI working condition. The multiproduct EPQ model considering backordering subject to the constraints of storage capacity, number of orders, and available budget was considered. The near optimal order quantities along with the maximum backorder levels of the products in a cycle are determined so that the total VMI inventory cost is minimized. A GA-based heuristic is proposed to solve the problem. Pasandideh et al. (2014b) present an integrated vendor-managed inventory model for a two-echelon supply chain organized as a single capacitated manufacturer at the first echelon and multiple retailers at the second echelon. Manufacturer produces different products whose demands are assumed decreasing functions of retail prices. A fair profit contract is designed for the manufacturer and the retailers and the problem is formulated into a bi-objective non-linear mathematical model. The lexicographic maxmin approach is utilized to obtain a fair non-dominated solution.

Sana (2014) develops a production-inventory model of a two-stage supply chain consisting of one manufacturer and one retailer to study production lot size/order quantity, reorder point sales teams' initiatives where the demand of the end customers is dependent on random variable and sales teams' initiatives simultaneously. The manufacturer produces the order quantity of the retailer at one lot in which the procurement cost per unit quantity follows a realistic convex function of production lot size. In the chain, the cost of sales team's initiatives/promotion efforts and wholesale price of the manufacturer are negotiated at the points such that their optimum profits reached nearer to their target profits. This study suggests to the management of firms to determine the optimal order quantity/production quantity, reorder point and sales teams' initiatives/promotional effort to achieve their maximum profits. An analytical method is applied to determine the optimal values of the decision variables. Finally, numerical examples with its graphical presentation and sensitivity analysis of the key 
parameters are presented to illustrate more insights of the model. In Sana et al. (2014), the replenishment size/production lot size problem both for perfect and imperfect quality products studied in their paper is motivated by the optimal strategy in a three-layer supply chain consisting of multiple suppliers, manufacturers and retailers. And each manufacturer produces each product with a combination of several raw materials which are supplied by each supplier. The defective products at suppliers and manufacturers are sent back to the respective upstream members at lower price than the respective purchasing price. Finally, the expected average profits of suppliers, manufacturers and retailers are formulated by trading off setup costs, purchasing costs, screening costs, production costs, inventory costs and selling prices. The objective of this chain is to compare between the collaborating system and Stakelberg game structure so that the expected average profit of the chain is maximized. In a numerical illustration, the optimal solution of the collaborating system shows a better optimal solution than the approach by Stakelberg. Thangam (2014) in their paper attempts to develop the retailer's inventory model with the effect of order cancellations during the advance sales period. The retailer announces a price discount program during advance sales period to promote his sales and also offers trade credit financing during the sales periods. The retailer availing trade credit period from his supplier offers a permissible delay period to his customers. The customer who gets an item is allowed to pay on or before the permissible delay period which is accounted from the buying time rather than from the start period of inventory sales. This accounts for significant changes in the calculations of interest payable and interest earned by the retailer. The retailer's total cost is minimized so as to find out the optimal replenishment cycle time and price discount policies through a solution procedure. The results derived in mathematical theorems are implemented in numerical examples, and sensitivity analyses on several inventory parameters are obtained.

\section{Notation and modeling}

The major notations used in this paper are as follows:

$n \quad$ Number of buyers

$a_{j} \quad$ Intercept of the demand curve of buyer $j$

$b_{j} \quad$ Slope of the demand curve of buyer $j$

$H b_{j} \quad$ Inventory holding cost of buyer $j$ at the independent mode (without VMI implementation)

Hs Inventory holding cost of the vendor (producer) at the independent mode

$H_{j_{\mathrm{VMI}}} \quad$ Resultant inventory holding cost of the integrated system of vendor and buyer $j$
$S b_{j} \quad$ Ordering (setup) cost of buyer $j$ at the independent mode

Ss Setup cost of the vendor per order at the independent mode

$S_{j_{\mathrm{VMI}}} \quad$ Continuously monitoring the stock status of buyer $j$ in VMI mode

$P \quad$ Total production rate of the vendor (producer)

$\theta_{j} \quad$ Flow cost per unit from producer to buyer $j$

$v_{j} \quad$ Transportation cost per unit delivered from vendor to buyer $j$

$\delta \quad$ Production cost per unit made by the vendor (producer)

$P D_{j} \quad$ Production and distribution cost of products to buyer $j$

$P R_{j} \quad$ Revenue share ratio between vendor and buyer $j$

$Q_{j} \quad$ Replenishment quantity for each buyer $j$

$W \quad$ Contract price between a vendor and a buyer

$W_{j} \quad$ Contract price between vendor and buyer $j$

$P_{j} \quad$ Production rate for buyer $j$ at the vendor's location

$y_{j} \quad$ Sales quantity of buyer $j$

$P\left(y_{j}\right) \quad$ Sales price of the product by buyer $j$ corresponding to sales quantity ' $y_{j}$ '

$P(y) \quad$ Sales price of the product

$y_{j_{\min }} \quad$ Minimum expected sales quantity of buyer $j$

$y_{j_{\max }} \quad$ Maximum expected sales quantity of buyer $j$

This paper investigates a TSPMBSC model operating under VMI mode.

\section{Description of the demand curve and contract price}

There are a lot of examples in practice in which each producer (vendor) has its own set of direct outlets (distribution centers/retailers addressed here as buyers). The major parameters of the corresponding models are: sales quantity ' $y$ ', the sales price at buyer's market ' $P(y)$ ', the contract price between the vendor and the buyer ' $W$ ' and the production rate for each buyer at vendor location. The sales quantity of the product at each location is highly influenced by its sales price and it depends on the factors such as the necessity of the commodity, the purchasing power of the customers, and the nature of the product (being perishable or storable). The general observation is that the higher the sales price, the lower is the sales quantity and vice versa. The relation between ' $P(y)$ ' and ' $y$ ' may be assumed to behave linearly and is given as (Nachiappan and Jawahar 2007):

$P(y)=a-b y$

where $a$ and $b$ represent the intercept and slope of the demand curve, respectively, as indicated in Fig. 1. Besides, sales quantity lies between a specific range between $y_{j_{\min }}$ and $y_{j_{\max }}$ and the validity of the linear demand assumption 


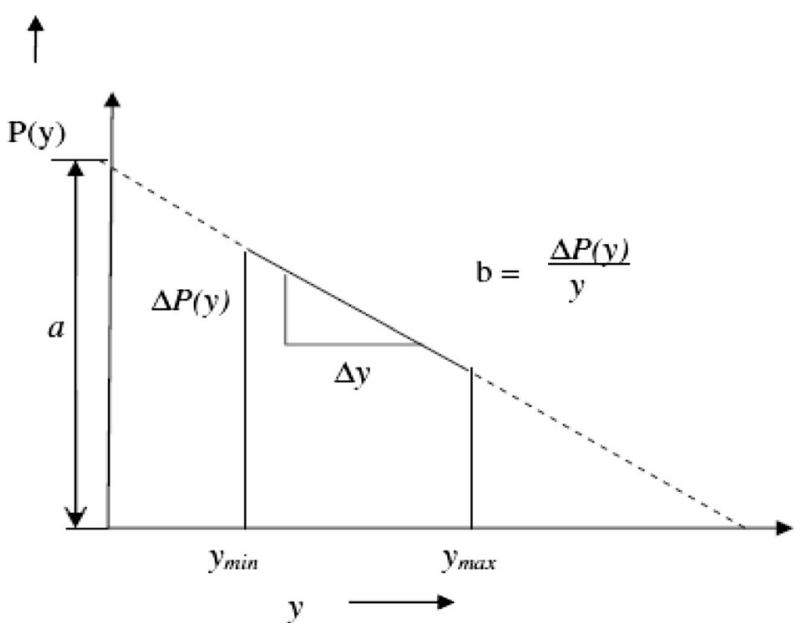

Fig. 1 Relation between the sales price and the sales quantity

function holds very well within this range. Since the buyers are not necessarily identical, the demand function of buyer $j$ may be stated as in (2)-(3)

$P\left(y_{j}\right)=a_{j}-b_{j} y_{j}$

s.t. $y_{j_{\min }} \leq y_{j} \leq y_{j_{\max }}$

A parameter which plays an important role on the profits of the both vendor and buyer(s) is the contract price. It is a price which is mutually agreed between the vendor and the buyer(s). Usually, it is assumed a value between the cost of manufacturing and the sales price. The nature of the product, the demand and the logistic cost play a critical role on determination of the contract price value. The commodities which have a good reputation and higher demand are usually fast moving and are involved with lower risk; in these circumstances, the buyer accepts the contract price closer to sales price. However, in other cases where the product is new and the demand is not yet stabilized, the contract price is expected being settled at a lower level, closer to the cost of manufacturing. In Nachiappan and Jawahar (2007), the contract price is a variable which is dependent on location, the competitiveness of the products, the production and the operational costs between vendor and buyer(s). The contract price between vendor and buyer $j$ is addressed by $W_{j}$.

\section{Vendor operations and costs}

Disney and Towill (2002) state that in VMI mode of cooperation among the members of a vendor-buyers chain, the vendor has more responsibility than the buyers and acts as a leader. The vendor monitors, manages and replenishes the inventory of all members (Achabal et al. 2000). The associated costs include production cost, distribution cost, order cost and stock holding cost. Production cost is derived from the expenses spent for producing a single unit ' $\delta$ ' and the aggregate demand ' $y$ ' (i.e., $y=\sum_{j=1}^{n} y_{j}$ ). Therefore, the total production cost can be stated as $\delta y$. The distribution cost is the multiplication of flow and transportation resource cost. The flow cost is the direct mileage and the carrier contract cost per unit of buyer $j$ ' $\theta_{j}$ ' and the transportation resource cost is the indirect cost such as mode of transport, human router cost and administrative costs and termed as ' $v_{j}$ ' per unit demand for the buyer $j$ (Dong and $\mathrm{Xu}$ 2002). Therefore, the distribution cost can be stated as ' $\theta_{j} y_{j} v_{j} y_{j}$ '. In this paper, it is assumed that the products to all locations are delivered by road and the value of ' $v_{j}$ ' is taken as 0.5 per unit as Dong and $\mathrm{Xu}$ (2002) consider. Therefore, the production and distribution costs ' $P D_{j}$ ' to the vendor for meeting sales ' $y_{j}$ ' of buyer $j$ can be given by (4)

$P D_{j}=\delta y_{j}+0.5 \theta_{j} y_{j}^{2}$

The vendor monitors the stock status and replenishes the stock. The buyer does not initiate orders. Therefore, the order cost per replenishment ' $S_{j_{\mathrm{VMI}}}$ ' associated with continuously monitoring the stock status is assumed as sum of the order cost of vendor ' $S s$ ' and order cost of buyer $j$ ' $S b_{j}$ ' (Nachiappan and Jawahar 2007) and is given as in (5)

$S_{j_{\mathrm{VMI}}}=S s+S b_{j}$

So, the cost involved with replenishing the batches ' $Q_{j}$ ' of demand of the buyer $j$ ' $y_{j}$ ' can be stated as ' $y_{j}\left(S s+S b_{j}\right)$ / $Q_{j}$. Nachiappan and Jawahar (2007) give this result in case where the vendor's production rate is infinite. However, this is also valid while the production rate is finite.

The inventory is held at both the vendor and the buyer(s) locations; the cost of holding one unit per unit time at vendor and buyer $j$ locations can be represented by ' $H s$ ' and ' $H b_{j}$ ', respectively. The vendor accumulates inventory before delivery to buyer ' $j$ '. As EPQ model, the vendor holds an average inventory of ' $Q_{j}\left(1-\frac{y_{j}}{P_{j}}\right) / 2$ ' to replenish buyer $j$. The inventory held at the vendor to replenish buyer $j$ is given to the buyer. The average inventory at the buyer location turns out to be ' $Q_{j}\left(1-\frac{y_{j}}{P_{j}}\right) / 2$ '; this is why the members use the VMI mode of cooperation. Therefore, in VMI mode, the cost of holding inventory ' $H_{j_{\mathrm{VMI}}}$ ' becomes the sum of the inventory holding cost at vendor and buyer (Nachiappan and Jawahar 2007); it can be given as in (6).

$H_{j_{\mathrm{VMI}}}=H s+H b_{j}$

The sum of the order cost and average inventory holding cost of the vendor for buyer ' $j$ ' namely ' $\mathrm{OSM}_{j}$ ', thus can be stated as in (7):

$\mathrm{OSM}_{j}=\left(S s+S b_{j}\right) y_{j} / Q_{j}+Q_{j}\left(H s+H b_{j}\right)\left(1-y_{j} / P_{j}\right) / 2$ 
Since the vendor produces for each buyer, assuming a common cycle time ' $T$ ' for different buyers, the common cycle time can be indicated as $T=\frac{Q_{j}}{y_{j}}, \forall j=1, \ldots, n$ (Silver et al. 1998). The sum of order and average inventory holding costs of the vendor for all buyers 'OSM' can be stated as in (8)

$\mathrm{OSM}=\sum_{j=1}^{n}\left[\left(S s+S b_{j}\right) / T+\left(H s+H b_{j}\right) \cdot T \cdot y_{j}\left(1-y_{j} / P_{j}\right) / 2\right]$,

where ' $T$ ' is computed as:

$T=\sqrt{\frac{2 \sum_{j=1}^{n}\left(S s+S b_{j}\right)}{\sum_{j=1}^{n} y_{j}\left(H s+H b_{j}\right)\left(1-y_{j} / P_{j}\right)}}$

The profit of the vendor when supplying the product to buyer $j$ ' $P V_{j}$ ' can be obtained from the difference between revenue to the vendor $\left(W_{j} y_{j}\right)$ and the total cost involved ' $P D_{j}+\mathrm{OSM}_{j}$ '. Therefore, the total profit to the vendor ' $P V$ ' by supplying its products to all the buyers can be obtained from (10)

$$
\begin{aligned}
P V= & \sum_{j=1}^{n}\left\{W_{j} y_{j}-\left(\delta y_{j}+0.5 \theta_{j} y_{j}^{2}\right)-\left[\left(S s+S b_{j}\right) / T\right.\right. \\
& \left.\left.+\left(H s+H b_{j}\right) \cdot T \cdot y_{j}\left(1-y_{j} / P_{j}\right) / 2\right]\right\}
\end{aligned}
$$

\section{Buyer operations and costs}

As Nachiappan and Jawahar (2007) declare, the costs associated with the buyers in VMI mode are the sales price and the contract price. The sales price for each buyer is determined using Eq. (2). The acceptable contract prices that would satisfy both the vendor and the buyer are derived from the revenue share ratio ' $P R_{j}$ '. Thus, the profit of buyer $j$ ' $P b_{j}$ ' in VMI mode is equal to the difference between the sales revenue and the cost of purchase as in (11).

$P b_{j}=P\left(y_{j}\right) y_{j}-W_{j} y_{j}=\left(a_{j}-b_{j} y_{j}\right) y_{j}-W_{j} y_{j}$

For a pre-specified value of revenue share ratio ' $P R_{j}=$ $P V_{j} / P b_{j}$ ' between the vendor and buyer $j$, the contract price can be stated as in (12).

\section{Objective function}

The objective function is considered as the maximization of channel profit of the supply chain. The mathematical expression of channel profit ' $P C$ ' can be stated as in (13).

$$
\begin{aligned}
P C= & P V+\sum_{j=1}^{n} P b_{j} \\
= & \sum_{j=1}^{n}\left\{a_{j} y_{j}-b_{j} y_{j}^{2}-\left(\delta y_{j}+0.5 \theta_{j} y_{j}^{2}\right)-\left[\left(S s+S b_{j}\right) / T\right.\right. \\
& \left.\left.+\left(H s+H b_{j}\right) \cdot T \cdot y_{j}\left(1-y_{j} / P_{j}\right) / 2\right]\right\}
\end{aligned}
$$

\section{Mathematical programming model}

The optimal or near optimal sales quantity and production rate for buyer $j$ namely ' $y_{j_{\mathrm{opt}}}$ ' and ' $P_{j_{\mathrm{opt}}}$ ' are obtainable from the following mathematical model which maximizes the channel profit namely ' $P C$ '.

$$
\begin{aligned}
\operatorname{Max} P C= & \sum_{j=1}^{n}\left\{a_{j} y_{j}-b_{j} y_{j}^{2}-\left(\delta y_{j}+0.5 \theta_{j} y_{j}^{2}\right)\right. \\
& \left.-\left[\left(S s+S b_{j}\right) / T+\left(H s+H b_{j}\right) \cdot T \cdot y_{j}\left(1-y_{j} / P_{j}\right) / 2\right]\right\}
\end{aligned}
$$

s.t $: y_{j_{\min }} \leq y_{j} \leq y_{j_{\max }}, \quad \forall j=1, \ldots, n$

$\sum_{j=1}^{n} P_{j}=P$

$y_{j} \leq P_{j}, \quad \forall j=1, \ldots, n$

$y_{j} \geq 0, \quad P_{j} \geq 0, \quad \forall j=1, \ldots, n$

Constraint (15) gives the valid upper and lower bounds of the sales quantity for the buyers. Constraint (16) guarantees that the sum of the buyer's production rates should be equal to the total production rate. Constraint (17) guarantees that the demand rate be less than the production rate for all the buyers as in EPQ model. Constraint (18) represents that the decision variables of the models should be non-negative. The optimal sales price ' $P\left(y_{j_{\text {opt }}}\right)$ ' can be obtained from (19).

$W_{j}=\frac{a_{j} y_{j} P R_{j}-b_{j} y_{j}^{2} P R_{j}+\delta y_{j}+0.5 \theta_{j} y_{j}^{2}+\left[\left(S s+S b_{j}\right) / T+\left(H s+H b_{j}\right) \cdot T \cdot y_{j}\left(1-y_{j} / P_{j}\right) / 2\right]}{\left(1+P R_{j}\right) y_{j}}$,

where ' $T$ ' is computed as in (9).

$P\left(y_{j_{\mathrm{opt}}}\right)=a_{j}-b_{j} y_{j_{\mathrm{opt}}}$ 
The acceptable contract price ' $W_{j_{\text {opt }}}$ ' is yielded by substituting the optimal sales quantity ' $y_{j_{\text {opt }}}$ ' in Eq. (12); the result is given as in (20). position); however, unlike GA, each potential solution is also assigned a randomized velocity and does not necessarily need to be encoded. Each individual or potential

$$
W_{j_{\mathrm{opt}}}=\frac{a_{j} y_{j_{\mathrm{opt}}} P R_{j}-b_{j} y_{j_{\mathrm{opt}}}^{2} P R_{j}+\delta y_{j_{\mathrm{opt}}}+0.5 \theta_{j} y_{j_{\mathrm{opt}}}^{2}+\left[\left(S s+S b_{j}\right) / T_{\mathrm{opt}}+\left(H s+H b_{j}\right) \cdot T_{\mathrm{opt}} \cdot y_{j_{\mathrm{opt}}}\left(1-y_{j_{\mathrm{opt}}} / P_{j_{\mathrm{opt}}}\right) / 2\right]}{\left(1+P R_{j}\right) y_{j_{\mathrm{opt}}}},
$$

where

$$
T_{\mathrm{opt}}=\sqrt{\frac{2 \sum_{j=1}^{n}\left(S s+S b_{j}\right)}{\sum_{j=1}^{n} y_{j_{\mathrm{opt}}}\left(H s+H b_{j}\right)\left(1-y_{j_{\mathrm{opt}}} / P_{j_{\mathrm{opt}}}\right)}}
$$

\section{Proposed heuristics}

\section{Particle swarm optimization}

Particle swarm optimization (PSO) was introduced by Kennedy and Eberhart (1995) as a population-based search algorithm. PSO is motivated from the simulation of social behavior of bird flocking. PSO uses a population of particles that fly through the search space to reach an optimum. Optimization with particle swarms has two major ingredients, the particle dynamics and the particle information exchange. The particle dynamics are derived from swarm simulations in computer graphics, and the information exchange component is inspired by social networks. These ingredients combine to make PSO a robust and efficient optimizer of real-valued objective functions (although PSO has also been successfully applied to combinatorial and discrete problems). PSO is accepted as a computational intelligent technique; the major difference between PSO and other well-known heuristics such as GA and SA is that the society members are aware of the other members' situation or at least of the best member and consider the obtained information in their decision making. Since the members can remember their best situation during the algorithm operations and always try to include this in their decision making, they can compensate immediately in case of a bad decision making. Each member can search the corresponding neighboring boundary without being worried about worsening the situation. The degree of being influenced by other members of the population is determined by a coefficient called learning coefficient.

PSO is similar to GA in that the system is initialized with a population of random solutions (called particle solution (i.e., particle) flies in the problem dimensional space with a velocity which is dynamically adjusted according to the flying experiences of its own and its colleagues. Each particle is affected by three factors: its own velocity, the best position it has achieved so far called ' $p$ best' and the overall best position achieved by all particles called 'gbest'. A particle changes its velocity based on the three addressed factors. Denoted by $n p$ the number of particles in the population (here, we assume $p=2 n)$, Let $X_{i}^{t}=\left[x_{i, 1}^{t}, x_{i, 2}^{t}, \ldots, x_{i, 2 n}^{t}\right]$ representing the position value of particle $i$ with respect to dimension $j$ $(j=1,2, \ldots, 2 n)$ at iteration $t$. We define the velocity of each particle as $V_{i}^{t}=\left[v_{i, 1}^{t}, v_{i, 2}^{t}, \ldots, v_{i, 2 n}^{t}\right]$ while each member of $v_{i}^{t}$ corresponds to each member of $X_{i}^{t}$. Let $P b_{i}^{t}=$ $\left[p b_{i, 1}^{t}, p b_{i, 2}^{t}, \ldots, p b_{i, 2 n}^{t}\right]$ be the best solution which particle $i$ has obtained by iteration $t$, and let $P_{g}^{t}=$ $\left[p_{g, 1}^{t}, p_{g, 2}^{t}, \ldots, p_{g, 2 n}^{t}\right]$ be the best solution obtained by iteration $t$.

Solution representation is one of the important steps while designing a PSO-based heuristic. The decision variables can be very good guidelines in this regard. In this paper, the solutions are represented as a string of $2 n$ characters in which the first $n$ characters represent the buyers' sales values and the second $n$ characters represent the production rates of the vendor as $\left\{\left(y_{1}, \ldots, y_{n}\right.\right.$, $\left.\left.p_{1}, \ldots, p_{n}\right) \mid y_{\min } \leq y_{i} \leq y_{\max }, y_{i} \leq p_{i}, \sum_{p i} \leq P\right\}$. Imagine that there are three buyers whose sales values are uniformly distributed as $y_{1} \sim U[1600,4800], y_{2} \sim U[700,1400]$, $y_{3} \sim U[1200,3600]$ and the production capacity of the vendor is as $P=18,000$. As the constraints of the model we should have $y_{i} \leq p_{i}, \sum_{i=1}^{3} p_{i} \leq 18,000$; the particle length should be $2 n=6$. Three random numbers should be generated corresponding to $y_{1}, y_{2}, y_{3}$ noting that $y_{\min }$ $\leq y_{i} \leq y_{\max }$. As the solutions are continuous, they will convert to the discrete solutions by random number generation in order to be usable in the problem. Table 1 illustrates a sample vector of particles $X_{i}^{t}$ used by PSO algorithm. 
Table 1 Solution representation of $X_{i}^{t}$

\begin{tabular}{lllllll}
\hline Dimension, $j$ & & & & & & \\
\hline Location & $y_{1}$ & $y_{2}$ & $y_{3}$ & $p_{1}$ & $p_{2}$ & $p_{3}$ \\
\hline$x_{i j}^{t}$ & 4620 & 1333 & 1505 & 5801 & 2296 & 2640 \\
\hline
\end{tabular}

We give a brief outline of the algorithm as:

\section{Step 1 Initialization}

- Set $t=0, n p=2 n$.

- Generate $n p$ particles randomly as explained before and make the vector of particles as $X_{i}^{0}=\left[y_{1}^{0}, \ldots, y_{n}^{0}, p_{1}^{0}, \ldots, p_{n}^{0}\right]$ where $y_{\min } \leq y_{i} \leq$ $y_{\max }, y_{i} \leq p_{i}$ and $\sum_{p i} \leq P$; the continuous values of the positions are generated randomly.

- Generate the initial velocities for each particle randomly, i.e., $\left\{V_{i}^{0}, \quad i=1,2, \ldots, N P\right\}$ where $V_{i}^{0}=\left[v_{i, 1}^{0}, v_{i, 2}^{0}, \ldots, v_{i, 2 n}^{0}\right]$. Initial velocities are generated using the formula $v_{i j}^{0}=v_{\min }+$ $\left(v_{\max }-v_{\min }\right) \times r$ where $v_{\min }=-4, v_{\max }=4$ and $r$ is a uniform random number between [0, 1]. Continuous velocity values are restricted as $v_{i j}^{t}=\left[v_{\min }, v_{\max }\right]=[-4,4], \quad$ where $\quad v_{\min }=$ $-v_{\max }$.

- Evaluate each particle in the swarm using the objective function $f_{i}^{0}\left(X_{i}^{0}\right)$ for $i=1,2, \ldots, n p$.

- For each particle in the swarm, set $P b_{i}^{0}=X_{i}^{0}$, where $P_{i}^{0}=\left[p b_{i, 1}^{0}=x_{i, 1}^{0}, p b_{i, 2}^{0}=x_{i, 2}^{0}, \ldots, p b_{i, 2 n}^{0}\right.$ $\left.=x_{i, 2 n}^{0}\right]$ together with its best fitness value, $f_{i}^{p b}$ for $i=1,2, \ldots, n p$.

- Find the best fitness value among the whole swarm such that $f_{l}=\min \left\{f_{i}^{0}\right\}$ for $i=1,2, \ldots$, $n p$ together with its corresponding positions $X_{l}^{0}$. Set global best to $G^{0}=X_{l}^{0}$ such that $G^{0}=\left[g_{1}=x_{l, 1}, \ldots, g_{\mathrm{DT}}=x_{l, 2 n}\right]$ with its fitness value $f^{g b}=f_{l}$.

Step 2 Update iteration counter, i.e., $t=t+1$

Step 3 Update inertia weight, i.e., $w^{t}=w^{t-1} \times \beta$ where $\beta$ is a decrement factor

Step 4 Update velocity, i.e., $v_{i j}^{t}=w^{t-1} v_{i j}^{t-1}+c_{1} r_{1}\left(p b_{i j}^{t-1}\right.$ $\left.-x_{i j}^{t-1}\right)+c_{2} r_{2}\left(g_{j}^{t-1}-x_{i j}^{t-1}\right)$, where $c_{1}$ and $c_{2}$ are acceleration coefficients and $r_{1}$ and $r_{2}$ are uniform random numbers between $[0,1]$

Step 5 Update positions, i.e., $x_{i j}^{t}=x_{i j}^{t-1}+v_{i j}^{t}$

Step 6 If $x_{i j}^{t}$ is continuous, round it to the nearest integer number in accordance with the model's constraints
Step 7 Update personal best; each particle is evaluated using the permutation to see if the personal best will improve. If $f_{i}^{t}<f_{i}^{p b}$ for $i=1,2, \ldots, n p$, then personal best is updated as $P_{i}^{t}=X_{i}^{t}$ and $f_{i}^{p b}=f_{i}^{t}$

Step 8 Update global best. Find the minimum value of personal best. That is, $f_{l}^{t}=\min \left\{f_{i}^{p b}\right\}$, for $i=$ $1,2, \ldots, n p ; l \in\{i ; i=1,2, \ldots, n p\}$. If $f_{i}^{t}<f_{i}^{g b}$, then the global best is updated as $G^{t}=X_{l}^{t}$ and $f^{g b}=f_{l}^{t}$

Step 9 Stopping criterion. If the number of iterations exceeds the maximum number of iterations, or maximum CPU time, then stop; otherwise go to step 2 .

\section{Genetic algorithm}

The proposed mathematical model for determining ' $y_{j_{\text {opt }}}$ ' and ' $P_{j_{\mathrm{opt}}}$ ' belongs to nonlinear integer programming (NIP) problem. A GA-based heuristic is proposed to evolve an optimal or near optimal sales quantity and production rate for buyer $j$, i.e., ' $y_{j_{\mathrm{opt}}}$ ' and ' $P_{j_{\mathrm{opt}}}$ ' to maximize the channel profit. The optimal sales price ' $P\left(y_{j_{\mathrm{opt}}}\right)$ ' and contract price ' $W_{j_{\mathrm{opt}}}$ ' are derived subsequently from the ' $y_{j_{\mathrm{opt}}}$ ' and ' $P_{j_{\mathrm{opt}}}$ '. GA as a population-based algorithm is a class of evolutionary algorithms. It is a generic optimization method, which can be applied to almost every problem. The feasible solutions of the problem are usually represented as strings of binary or real numbers called chromosomes. Each chromosome has a fitness value, which corresponds to the objective function value of the model. Initially, there is a population of chromosomes randomly generated; then, a number of chromosomes are selected as parents for mating to produce new chromosomes (i.e., solutions) called offspring. The mating of parents is carried out applying a few GA operators, such as crossover and mutation. The selection of parents and producing offspring are repeated until a stopping rule (e.g., elapsing a certain number of iterations) is satisfied (Goldberg 1989). Before giving a general outline of the proposed GA-based heuristic, a few additional notations are defined:

Pop_size Size of the population of solutions that remains constant during the algorithm performance.

Max_iteration Number of generations produced until the algorithm stops.

$p_{\mathrm{c}}$ Crossover rate, which is the probability of selecting a chromosome in each generation for crossover.

$p_{\mathrm{m}}$ Mutation rate, which is the probability of selecting a gene or bit inside a chromosome for mutating. 
Fitness_function Fitness function value, which exactly corresponds to the objective function value in this paper.

We give a brief outline of the algorithm as:

Step 1 Initialization

- Set Pop_size, Max_iteration, $p_{\mathrm{c}}$ and $p_{\mathrm{m}}$.

Step 2 Randomly generate the initial population

Step 3 Repeat until Max_iteration:

Step 3.1 Perform the reproduction operator according to the roulette wheel rule to make a new population

Step 3.2 Select the parent chromosomes from the obtained population, each with probability $p_{\mathrm{c}}$

Step 3.3 Crossover:

(a) Determine the pairs of parents among the parent chromosomes.

(b) Apply the crossover operator to produce two offspring corresponding to each pair.

(c) Replace each offspring in the population instead of the parents.

Step 3.4 Apply the mutation operator on the population with probability $p_{\mathrm{m}}$

Step 3.5 Calculate Fitness_function for each chromosome and save the best value in $b v$ (best value)

\section{Step 4 Print $b v$}

Each chromosome consists of $2 n$ genes. The first $n$ genes represent the sales quantities of the buyers and the second $n$ genes represent the production rates of them. As an example, the chromosome [ $\left.\begin{array}{llllllll}1 & 1 & 3 & 4 & 5 & 7 & 9 & 10\end{array}\right]$ indicates that there are four buyers whose sales quantities are 1, 1, 3 and 4, respectively, and production rates are 5, 7, 9 and 10, respectively.

The Pop_size, $p_{\mathrm{c}}$ and $p_{\mathrm{m}}$ are determined through the try and error method while Max_iteration is assumed equal to 2000.

\section{Simulated annealing}

SA proposed by Kirkpatrick et al. (1983) is a stochastic and neighborhood-based search algorithm motivated from an analogy between the simulation of the annealing of solids and the strategy of solving combinatorial optimization problems. SA has been widely applied to solve combinatorial optimization problems as Yao (1995) declares. It is inspired by the physical process of heating a substance and then slowly cooling it, until a strong crystalline structure to be formed. This process is simulated through gradually lowering an initial temperature until the system reaches an equilibrium point so that no more changes occur. Generally, details of SA proposed are as follows:

Algorithm: simulated annealing

1: Initialize parameters $T_{0}, N, K, \alpha, T_{f}$

2: Initialized counter $n=0, k=0$

3: Do (outside loop)

4: Set $n=0$

5: Generate initial solution $X^{0}$ : Set $\quad X^{\text {Best }}=X^{0}$

6: Do (inside loop)

7: Generate neighboring solution $X^{n-1}$ by operation $\left(X_{n} \rightarrow X^{n-1}\right)$

8: If $f\left(X^{n+1}\right) \prec f\left(X^{n}\right)$ then

9: $X^{n}=X^{n+1}:$ Set $n=n+1$

10: Else

11: Generate random Rand $\rightarrow u(0,1)$

12: If Rand $<\mathrm{e}^{-\Delta F / T_{k}}$ then $X^{n}=X^{n+1}$ : set $n=n+1$

13: End if

14: Update $X^{\text {Best }}$

15: Loop until $(n \leq N)$

16: $T_{r+1}=\alpha T_{r}$

17: Loop until frozen

\section{Computational experiments}

Nachiappan and Jawahar (2007) analyzed their proposed model and methodology by a case study carried out at the SNP dairy company located in Madurai, India. The dairy manufacturer (vendor) supplies its product (milk packets) to the customers at different locations (buyers). Since the structure of our proposed model is near to that of Nachiappan and Jawahar (2007), we have provided a few numerical problems inspiring from those of Nachiappan and Jawahar (2007). The numerical problems are given in three categories while considering three, five, and eight buyers in the model. Since the number of parameters is too much, the buyer-related parameters are considered fixed, while the vendor-related parameters are changed in order to do the sensitivity analysis. The values of the buyer-related parameters are given in Table 2. Five problems are selected from each category as small, medium and large size 
Table 2 Values of the buyer-related parameters

\begin{tabular}{|c|c|c|c|c|c|c|c|c|c|c|c|c|c|c|c|c|}
\hline \multirow[b]{2}{*}{$j$} & \multicolumn{3}{|c|}{ Buyer-related data for $n=3$} & \multicolumn{5}{|c|}{ Buyer-related data for $n=5$} & \multicolumn{8}{|c|}{ Buyer-related data for $n=8$} \\
\hline & 1 & 2 & 3 & 1 & 2 & 3 & 4 & 5 & 1 & 2 & 3 & 4 & 5 & 6 & 7 & 8 \\
\hline$H b_{j}$ & 8 & 10 & 10 & 8 & 10 & 10 & 6 & 7 & 8 & 10 & 10 & 6 & 7 & 12 & 13 & 14 \\
\hline$S b_{j}$ & 24 & 11 & 29 & 24 & 11 & 29 & 14 & 25 & 24 & 11 & 29 & 14 & 25 & 12 & 30 & 22 \\
\hline$a_{j}$ & 31 & 35 & 37 & 31 & 35 & 37 & 32 & 39 & 31 & 35 & 37 & 32 & 39 & 33 & 36 & 38 \\
\hline$b_{j}$ & 0.008 & 0.004 & 0.006 & 0.008 & 0.004 & 0.006 & 0.003 & 0.004 & 0.008 & 0.004 & 0.006 & 0.003 & 0.004 & 0.005 & 0.007 & 0.006 \\
\hline$y_{j_{\min }}$ & 1600 & 700 & 1200 & 1600 & 700 & 1200 & 1500 & 900 & 1600 & 700 & 1200 & 1500 & 900 & 700 & 800 & 1200 \\
\hline$y_{j_{\max }}$ & 4800 & 1400 & 3600 & 4800 & 1400 & 3600 & 3000 & 2700 & 4800 & 1400 & 3600 & 3000 & 2700 & 3500 & 4900 & 3000 \\
\hline$\theta_{j}$ & 0.004 & 0.008 & 0.005 & 0.004 & 0.008 & 0.005 & 0.005 & 0.007 & 0.004 & 0.008 & 0.005 & 0.005 & 0.007 & 0.005 & 0.007 & 0.006 \\
\hline
\end{tabular}

Table 3 Values of the vendor-related parameters

\begin{tabular}{lrrrl}
\hline Level & $H s$ & $S s$ & \multicolumn{1}{c}{$\delta$} & $P$ \\
\hline Low (-1) & 3 & 5 & 5 & 18,000 \\
Up (+1) & 15 & 40 & 10 & 27,000 \\
\hline
\end{tabular}

problems. The values of the vendor-related parameters are given in Table 3.

In the rest of this section, we are going to compare the proposed GA, discrete particle swarm optimization algorithm (DPSO), and SA for TSVMBSC problem. We have also used LINGO solver to assess the performance of the proposed heuristics. All the heuristics are coded in Matlab7.0 software and run on a PC with $1.67 \mathrm{GHz}$ processor (Intel Pentium 4), 256 MG memory and windows XP Professional Operating System.

We have used relative percentage index (RPI) to assess the performance of the proposed heuristics. This index is one of the well-known indexes in this regard for single objective problems. We have solved a number of instances for each numerical problem. RPI can be computed by Eq. (22) in which Max $_{\text {sol }}$ and Worst ${ }_{\text {sol }}$ represent the best and worst objective function values obtained from solving the instances of each numerical problem while solving it by different heuristics; $A \lg _{\text {sol }}$ represents the objective function value for each instance of a numerical problem.

$\mathrm{RPI}=\frac{\operatorname{Max}_{\mathrm{sol}}-A \lg _{\mathrm{sol}}}{\operatorname{Max}_{\mathrm{sol}}-\text { Worst }_{\mathrm{sol}}}$

RPI can take values between 0 and 1 . Clearly, lower values of RPI are preferred. Table 4 gives the RPI values for each numerical problem while solving it by each heuristic as well as using LINGO solver. We have considered the number of instances for each numerical problem equal to five times; the average of the obtained objective function values from solving the five instances is considered as $\overline{\mathrm{RPI}}$ for each numerical problem with respect to each heuristic.
The CPU times are considered the same for the heuristics; however, we have reported the CPU times when each algorithm reached the best corresponding solution.

As it is clear from Table 4, the RPI for DPSO is less than that of other heuristics; however, the average CPU time of LINGO solver is less than that of other heuristics. We have also used statistical $t$ test at significance level $\alpha=0.05$ to compare each heuristic with the other considering $H_{0}: D=0$ against $H_{1}: D>0$ in which $D$ represents the difference between the average of $\overline{\mathrm{RPI}}$ of the first heuristic and that of the second. Therefore, hypothesis $H_{0}$ can be rejected if and only if $t=\frac{\bar{D}}{S_{D} / \sqrt{n}}>t_{\alpha ; n-1}$. Table 5 illustrates the results.

Figure 2 indicates the average value of LSD with confidence interval $95 \%$ for various heuristics. It is clear from Fig. 2 that DPSO is superior compared with other heuristics and LINGO solver.

\section{Conclusions and suggestion}

This paper presents a TSPMBSC model under the VMI mode of operation. It is the extension of Nachiappan and Jawahar (2007) for the case where the vendor (producer) replenishes orders as EPQ, i.e., the product gradually enters into the vendor's location. The final model can be stated as a mathematical programming model with the objective function of channel profit and the two decision variables of sales quantity and production rate. Thereafter, the optimal values of the decision variables are determined. The aforementioned problem is NP-hard which means too difficult to be solved during a logical amount of time. We presented a DPSObased heuristic to solve the problem. To prove the efficiency of the proposed heuristic, two distinct kinds of heuristics were used, including innovative searching method of the GA, and SA; however, LINGO solver was also used. The heuristics applied to solve a set of small, medium and large 
Table 4 Average relative percentage deviation $(\overline{\mathrm{RPI}})$ and average CPU time for algorithms

\begin{tabular}{|c|c|c|c|c|c|c|c|c|}
\hline \multirow[t]{3}{*}{ Problem size } & \multicolumn{8}{|c|}{ Comparative algorithms } \\
\hline & \multicolumn{2}{|l|}{ GA } & \multicolumn{2}{|l|}{ SA } & \multicolumn{2}{|l|}{ DPSO } & \multicolumn{2}{|l|}{ LINGO } \\
\hline & $\overline{\mathrm{RPI}}$ & CPU time & $\overline{\mathrm{RPI}}$ & CPU time & $\overline{\mathrm{RPI}}$ & CPU time & $\overline{\mathrm{RPI}}$ & CPU time \\
\hline \multicolumn{9}{|c|}{ Small problem } \\
\hline PS1 & 0.0000 & 0.30 & 0.0000 & 0.27 & 0.0000 & 0.26 & 0.0000 & 0.27 \\
\hline PS2 & 0.2000 & 2.08 & 0.2000 & 1.87 & 0.1000 & 1.59 & 0.0000 & 1.62 \\
\hline PS3 & 0.2000 & 1.87 & 0.2000 & 1.69 & 0.2000 & 1.39 & 0.0000 & 1.41 \\
\hline PS4 & 0.4000 & 5.74 & 0.2000 & 4.74 & 0.2000 & 4.37 & 0.0000 & 4.33 \\
\hline PS5 & 0.2000 & 6.23 & 0.1333 & 6.16 & 0.0667 & 5.20 & 0.0000 & 5.15 \\
\hline \multicolumn{9}{|c|}{ Medium problems } \\
\hline PM1 & 0.8667 & 16.33 & 0.7000 & 14.26 & 0.6000 & 15.52 & 0.5000 & 15.91 \\
\hline PM2 & 0.3030 & 37.21 & 0.2017 & 32.02 & 0.1517 & 34.65 & 0.1617 & 33.12 \\
\hline PM3 & 0.7750 & 37.18 & 0.5002 & 32.24 & 0.4379 & 31.87 & 0.5128 & 30.91 \\
\hline PM4 & 0.3715 & 32.67 & 0.3004 & 29.65 & 0.2307 & 24.42 & 0.5081 & 24.42 \\
\hline PM5 & 0.4806 & 35.55 & 0.4444 & 26.80 & 0.5833 & 27.82 & 0.8056 & 28.43 \\
\hline \multicolumn{9}{|c|}{ Large problems } \\
\hline PL1 & 0.1044 & 478.28 & 0.3116 & 478.28 & 0.2889 & 404.75 & 0.3864 & 389.78 \\
\hline PL2 & 0.3667 & 448.07 & 0.3833 & 448.07 & 0.3750 & 437.87 & 0.6333 & 433.87 \\
\hline PL3 & 0.5331 & 352.03 & 0.6662 & 352.03 & 0.5996 & 309.00 & 0.9975 & 293.93 \\
\hline PL4 & 0.0333 & 539.38 & 0.1833 & 539.38 & 0.1778 & 513.78 & 0.2833 & 513.78 \\
\hline PL5 & 0.7273 & 935.95 & 0.8473 & 935.95 & 0.6909 & 832.05 & 0.9818 & 832.05 \\
\hline Average & 0.371 & 195.26 & 0.351 & 193.56 & 0.313 & 186.30 & 0.385 & 173.93 \\
\hline
\end{tabular}

Table 5 Results from $t$ test for the $\overline{\mathrm{RPI}}$

\begin{tabular}{lrl}
\hline Algorithms & \multicolumn{1}{l}{$t$} & $P$ value \\
\hline DPSO: LINGO & -0.93 & 0.717 \\
DPSO: GA & -1.89 & 0.853 \\
DPSO: SA & -1.48 & 0.802 \\
GA: SA & 1.40 & $0.173^{*}$ \\
SA: LINGO & -0.67 & 0.476 \\
GA: LINGO & 1.90 & $0.113^{*}$ \\
\hline
\end{tabular}

* Means that the difference is significant, i.e., $P$ value $<\alpha$

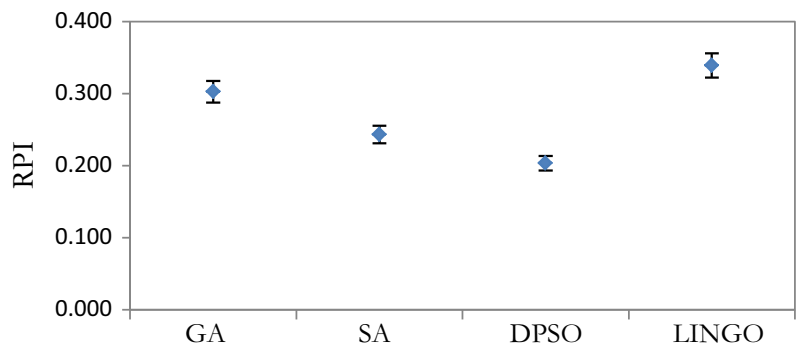

Fig. 2 Plot of $\overline{\text { RPI }}$ for the type of algorithm factor

size problems. The results indicated that the DPSO excels compared to the other rival heuristics.

Though the model considered in this paper is restricted to two-echelons, further analysis is required to study the performance under multi-echelon supply chains. Besides, demand can be lost or backordered while it is stochastic.

Open Access This article is distributed under the terms of the Creative Commons Attribution 4.0 International License (http://crea tivecommons.org/licenses/by/4.0/), which permits unrestricted use, distribution, and reproduction in any medium, provided you give appropriate credit to the original author(s) and the source, provide a link to the Creative Commons license, and indicate if changes were made.

\section{References}

Achabal DD, Mcintyre SH, Smith SA, Kalyanam K (2000) A decision support system for VMI. J Retail 76(4):430-454

Almehdawe E, Mantin B (2010) Vendor managed inventory with a capacitated manufacturer and multiple retailers: retailer versus manufacturer leadership. Int J Prod Econ 128(1):292-302

AriaNezhad MG, Makuieand A, Khayatmoghadam S (2013) Developing and solving two-echelon inventory system for perishable items in a supply chain: case study (Mashhad Behrouz Company). J Ind Eng Int. doi:10.1186/2251-712X-9-39

Bhattacharjee S, Ramesh R (2000) A multi-period profit maximizing model for retail supply chain management: an integration of demand and supply side mechanism. Eur J Oper Res 122:584-601

Bichescu BC, Fry MJ (2009) Vendor-managed inventory and the effect of channel power. OR Spectr 31(1):195-228

Bookbinder JH, Gümüş M, Jewkes EM (2010) Calculating the benefits of vendor managed inventory in a manufacturer-retailer system. Int J Prod Res 48(19):5549-5571 
Cárdenas-Barrón LE, Treviño-Garza G, Wee HM (2012) A simple and better algorithm to solve the vendor managed inventory control system of multi-product multi-constraint economic order quantity model. Expert Syst Appl 39(3):3888-3895

Costa L, Oliveira P (2001) Evolutionary algorithms approach to the solution of mixed integer nonlinear programming problems. Comput Chem Eng 25:257-266

Darwish MA, Odah OM (2010) Vendor managed inventory model for single-vendor multi-retailer supply chains. Eur J Oper Res 204(3):473-484

Diabat A (2014) Hybrid algorithm for a vendor managed inventory system in a two-echelon supply chain. Eur J Oper Res 238(1):114-121

Disney SM, Towill DR (2002) A procedure for optimization of the dynamic response of a vendor managed inventory systems. Comput Ind Eng 43:27-58

Dong Y, Xu K (2002) A supply chain model of vendor managed inventory. Transp Res Part E Logist Transp Rev 38(2):75-95

Goh S-A, Ponnambalam SG (2010) A particle swarm optimization algorithm for optimal operating parameters of VMI systems in a two-echelon supply chain. Swarm Evolut Memet Comput 6466:440-447

Goh S-A, Ponnambalam SG, Jawahar N (2012) Evolutionary algorithms for optimal operating parameters of vendor managed inventory systems in a two-echelon supply chain. Adv Eng Softw 52:47-54

Goldberg DE (1989) Genetic algorithms in search, optimisation and machine learning. Addision Wesley, Reading

Govindan K (2013) Vendor-managed inventory: a review based on dimensions. Int J Prod Res 51(13):3808-3835

Grieger M (2003) Electronic marketplaces: a literature review and a call for supply chain management research. Eur J Oper Res 144:280-294

Guan R, Zhao X (2010) On contracts for VMI program with continuous review (r, Q) policy. Eur J Oper Res 207(2):656-667

Kennedy J, Eberhart RC (1995) Particle swarm optimization. In: Proceedings of IEEE international conference on neural networks, IV, pp 1942-1948

Kirkpatrick S, Gelatt CD, Vecchi MP (1983) Optimization by simulated annealing. Science New Ser 220:671-680

Lau AHL, Lau HS (2003) Effects of a demand curve's shape on the optimal solutions of a multi echelon inventory/pricing model. Eur J Oper Res 147:530-548

Lu L (1995) A one-vendor multi-buyer integrated inventory model. Eur J Oper Res 81(2):312-323

Maloni MJ, Benton WC (1997) Supply chain partnership: opportunities for operations research. Eur J Oper Res 101:419-429

Nachiappan SP, Jawahar N (2007) A genetic algorithm for optimal operating parameters of VMI system in a two-echelon supply chain. Eur J Oper Res 182(3):1433-1452

Nia AR, Far MH, Niaki STA (2013) A fuzzy vendor managed inventory of multi-item economic order quantity model under shortage: an ant colony optimization algorithm. Int J Prod Econ 155:259-271

Pal B, Sana SS, Chaudhuri K (2012a) A three layer multi-item production-inventory model for multiple suppliers and retailers. Econ Model 29(6):2704-2710

Pal B, Sana SS, Chaudhuri K (2012b) A multi-echelon supply chain model for reworkable items in multiple-markets with supply disruption. Econ Model 29(5):1891-1898

Pasandideh SHR, Niaki STA, Nia AR (2010) An investigation of vendor-managed inventory application in supply chain: the EOQ model with shortage. Int J Adv Manuf Technol 49(1-4):329-339

Pasandideh SHR, Niaki STA, Nia AR (2011) A genetic algorithm for vendor managed inventory control system of multi-product multi-constraint economic order quantity model. Expert Syst Appl 38(3):2708-2716

Pasandideh SHR, Niaki STA, Far MH (2014a) Optimization of vendor managed inventory of multiproduct EPQ model with multiple constraints using genetic algorithm. Int J Adv Manuf Technol 71(1-4):365-376

Pasandideh SHR, Niaki STA, Niknamfar AM (2014b) Lexicographic max-min approach for an integrated vendor-managed inventory problem. Knowl Based Syst 59:58-65

Rad RH, Razmi J, Sangari MS, Ebrahimi ZF (2014) Optimizing an integrated vendor-managed inventory system for a single-vendor two-buyer supply chain with determining weighting factor for vendor's ordering cost. Int J Prod Econ 153:295-308

Razmi J, Rad RH, Sangari MS (2010) Developing a two-echelon mathematical model for a vendor-managed inventory (VMI) system. Int J Adv Manuf Technol 48(5-8):773-783

Sadeghi J, Mousavi SM, Niaki STA, Sadeghi S (2013) Optimizing a multi-vendor multi-retailer vendor managed inventory problem: two tuned meta-heuristic algorithms. Knowl Based Syst 50:159-170

Sana SS (2014) Optimal production lot size and reorder point of a two-stage supply chain while random demand is sensitive with sales teams' initiatives. Int J Syst Sci. doi:10.1080/00207721. 2014.886748

Sana SS, Chedid JA, Navarro KS (2011) A production-inventory model of imperfect quality products in a three-layer supply chain. Decis Support Syst 50(2):539-547

Sana SS, Chedid IA, Navarro KS (2014) A three layer supply chain model with multiple suppliers, manufacturers and retailers for multiple items. Appl Math Comput 229:139-150

Shao H, Li Y, Zhao D (2011) An optimal dicisional model in twoechelon supply chain. Procedia Eng 15:4282-4286

Silver AE, Pyke DF, Peterson R (1998) Inventory management and production planning and scheduling. Wiley, London

Taleizadeh AA, Noori-daryan M (2014) Pricing, manufacturing and inventory policies for raw material in a three-level supply chain. Int J Syst Sci. doi:10.1080/00207721.2014.909544

Thangam A (2014) Retailer's inventory system in a two-level trade credit financing with selling price discount and partial order cancellations. J Ind Eng Int. doi:10.1186/2251-712X-10-3

Toptal A, Çetinkaya S (2008) Quantifying the value of buyer-vendor coordination: analytical and numerical results under different replenishment cost structures. Eur J Oper Res 187(3):785-805

Van der Vlist P, Kuik R, Verheijen B (2007) Note on supply chain integration in vendor managed inventory. Decis Support Syst 44(1):360-365

Verma NK, Chakraborty A, Chatterjee AK (2014) Joint replenishment of multi retailer with variable replenishment cycle under VMI. Eur J Oper Res 233(3):787-789

Waller M, Johnson ME, Davis T (2001) Vendor managed inventory in the retail supply chain. J Bus Logist 20(1):183-203

Wang CX (2009) Random yield and uncertain demand in decentralized supply chains under the traditional and VMI arrangements. Int J Prod Res 47(7):1955-1968

Wang W-T, Wee H-M, Tsao H-SJ (2010) Revisiting the note on supply chain integration in vendor-managed inventory. Decis Support Syst 48(2):419-420

Wong WK, Qi J, Leung SYS (2009) Coordinating supply chains with sales rebate contracts and vendor-managed inventory. Int J Prod Econ 120(1):151-161

Yao X (1995) A new simulated annealing algorithm. Int J Comput Math 56:161-168

Yao MJ, Chiou CC (2004) On a replenishment coordination model in an integrated supply chain with one vendor and multiple buyers. Eur J Oper Res 159:406-419 
Yao Y, Evers PT, Dresner ME (2007) Supply chain integration in vendor-managed inventory. Decis Support Syst 43:663-674

Yu Y, Chu F, Chen H (2009a) A Stackelberg game and its improvement in a VMI system with a manufacturing vendor. Eur J Oper Res 192(3):929-948

Yu Y, Huang GQ, Liang L (2009b) Stackelberg game-theoretic model for optimizing advertising, pricing and inventory policies in vendor managed inventory (VMI) production supply chains. Comput Ind Eng 57(1):368-382
Zavanella L, Zanoni S (2009) A one-vendor multi-buyer integrated production-inventory model: the 'Consignment Stock' case. Int J Prod Econ 118(1):225-232

Zhang T, Liang L, Yu Y, Yu Y (2007) An integrated vendor-managed inventory model for a two-echelon system with order cost reduction. Int J Prod Econ 109:241-253 